\title{
Occult intraocular foreign body masquerading as panuveitis: inductively coupled mass spectrometry and electrophysiologic analysis
}

\author{
Steven Yeh • Martina Ralle • Isabella T. Phan • \\ Peter J. Francis • James T. Rosenbaum • \\ Christina Joy Flaxel
}

Received: 1 July 2011 / Accepted: 19 July 2011 /Published online: 31 July 2011

(C) The Author(s) 2011. This article is published with open access at Springerlink.com

\section{Introduction}

Retained intraocular foreign bodies (IOFB) may rarely present as chronic uveitis, either with isolated anterior uveitis or with posterior segment inflammation. The heavy metals copper and iron may lead to both acute and chronic inflammatory processes resulting in severe inflammation, retinal degeneration, and resultant visual loss. Penetrating or perforating ocular injuries are easily recognizable in the majority of cases; however, a high index of suspicion is necessary to recognize an occult penetrating injury because of the severe visual impairment that can ensue. We describe an unusual case of a retained IOFB, which presented 6 months after a probable occult penetrating injury from metallic IOFB trauma. The severity of the patient's inflammation on presentation and retinal findings in the

S. Yeh

Emory Eye Center, Emory University School of Medicine,

Atlanta, GA, USA

M. Ralle

Department of Biochemistry and Molecular Biology, Oregon Health and Science University,

Portland, OR, USA

\section{T. Phan}

Department of Ophthalmology, University of California San Francisco School of Medicine,

San Francisco, CA, USA

P. J. Francis · J. T. Rosenbaum · C. J. Flaxel $(\square)$

Casey Eye Institute, Oregon Health and Science University, 3375 SW Terwilliger Blvd.,

Portland 97239-4197 OR, USA

e-mail: flaxelc@ohsu.edu contralateral eye prompted further diagnostic testing including inductively coupled mass spectrometry of the IOFB and electrophysiologic testing. This rare clinical scenario, laboratory findings, and therapeutic considerations are presented.

\section{Case report}

A 19-year-old Caucasian male was referred to the Casey Eye Institute with a 3-week history of decreased vision and photophobia involving the right eye (OD). He reported hammering metal on metal 6 months prior and felt a foreign object enter his eye. The patient was evaluated at an urgent care facility where his eye was flushed, and tobramycin-dexamethasone ointment was prescribed. The patient's foreign body sensation improved, and he remained asymptomatic until 3 weeks prior to our evaluation. After ophthalmologic evaluation by another physician, he was referred to our service for suspicion of an occult IOFB.

Visual acuities were 20/50 OD and 20/20 in the left eye (OS). Slit lamp examination showed trace anterior chamber cell and 2+ vitreous cell OD and trace anterior chamber cell OS. Dilated funduscopic examination (DFE) OD showed mild optic disc edema, retinal vascular tortuosity, and a white elevated mass inferotemporally with surrounding pigmentary mottling and an overlying vitreous condensation (Fig. 1). DFE OS showed a nerve fiber layer hemorrhage along the superotemporal arcade. A B-scan ultrasound confirmed a metallic IOFB and orbital CT scan revealed metallic artifact from the IOFB with a possible posterior rupture (Fig. 2). No other foreign bodies were identified. 

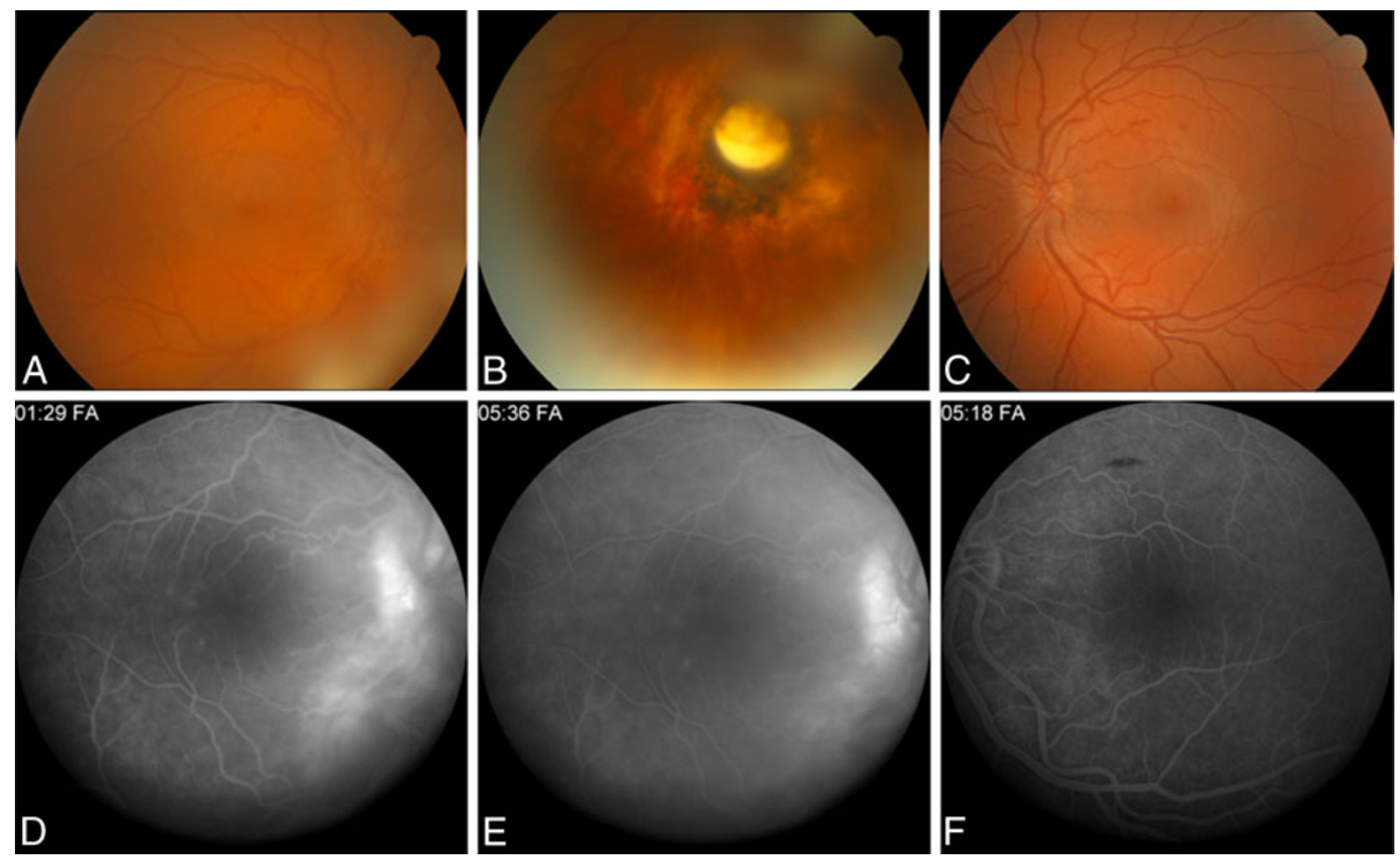

Fig. 1 Fundus photograph of the right eye at the patient's initial presentation showed mild vitreous haze, optic disc edema, and retinal vascular tortuosity (a) with an inferotemporally located yellow-white, well-circumscribed mass with surrounding chorioretinal hyperpigmentation and atrophy (b). c Fundus photograph of the left eye shows

\section{Surgical technique}

Pars plana vitrectomy (PPV) and IOFB extraction were planned for the presumptively encapsulated IOFB. A standard three-port 20-gauge PPV was performed, taking care to dissect all of the vitreoretinal adhesions extending from the apex of the capsule surrounding the IOFB. Following vitreous dissection around the IOFB, alligator forceps were used to free the IOFB and its fibrotic capsule from the retina. Because of the dense adhesions completely surrounding the IOFB, additional dissection with the vitreous cutter was necessary to free the fibrotic adhesions extending from the IOFB capsule to the retina. After successfully liberating the IOFB from the retina, the superotemporal sclerotomy was enlarged with a microvitreoretinal blade and the IOFB was removed from the eye with alligator forceps. Next, 7-0 vicryl suture was used to close the temporal aspect of the superotemporal sclerotomy site to address the large retinal defect and associated inferotemporal retinal detachment, which were observed. The retinal defect was marked with endodiathermy; airfluid exchange was performed, and the break was surrounded with endolaser. A 276-tire supporting the inferior 180-degree scleral bed, 240-band, and 70-Watzke sleeve secured with 5-0 Mersilene horizontal mattress focal intraretinal hemorrhage within the superotemporal arcade. d, e A fluorescein angiography showed leakage from the optic nerve, peripapillary retinal vessels, and mild leakage in the parafoveal region. f Fluorescein angiography of the left eye showed no optic disc or vascular leakage

sutures were used to provide additional support to the inferior retinal defect. Twenty percent SF6 gas was exchanged for air, and the sclerotomy and conjunctiva were closed in standard fashion.

\section{Metallurgic analysis}

Sample preparation

Of the ferromagnetic IOFB, $4.0 \mathrm{mg}$ was added to $1.5 \mathrm{ml}$ of aqua regia (3:1 parts $\mathrm{HNO}_{3}: \mathrm{HCl}$, Fisher, Metals Grade) and heated under reflux for $3 \mathrm{~h}$ during which the metal fragment completely dissolved. The resulting bright-yellow solution was brought up to exactly $10 \mathrm{ml}$ with $1 \% \mathrm{HNO}_{3}$. The solution was diluted into $1 \% \mathrm{HNO}_{3}$ to fall within the range of the standard curve (the sample was measured a total of four times at dilutions of $100 \times, 1,000 \times, 100 \times$, and $2,000 \times$ ).

Inductively coupled plasma mass spectrometry analysis

Inductively coupled plasma mass spectrometry (ICP-MS) analysis was performed using an Agilent $7700 \times$ system equipped with an ASX-250 Autosampler. The system was operated at a radio frequency power of $1,550 \mathrm{~W}$, an $\operatorname{argon}$ 

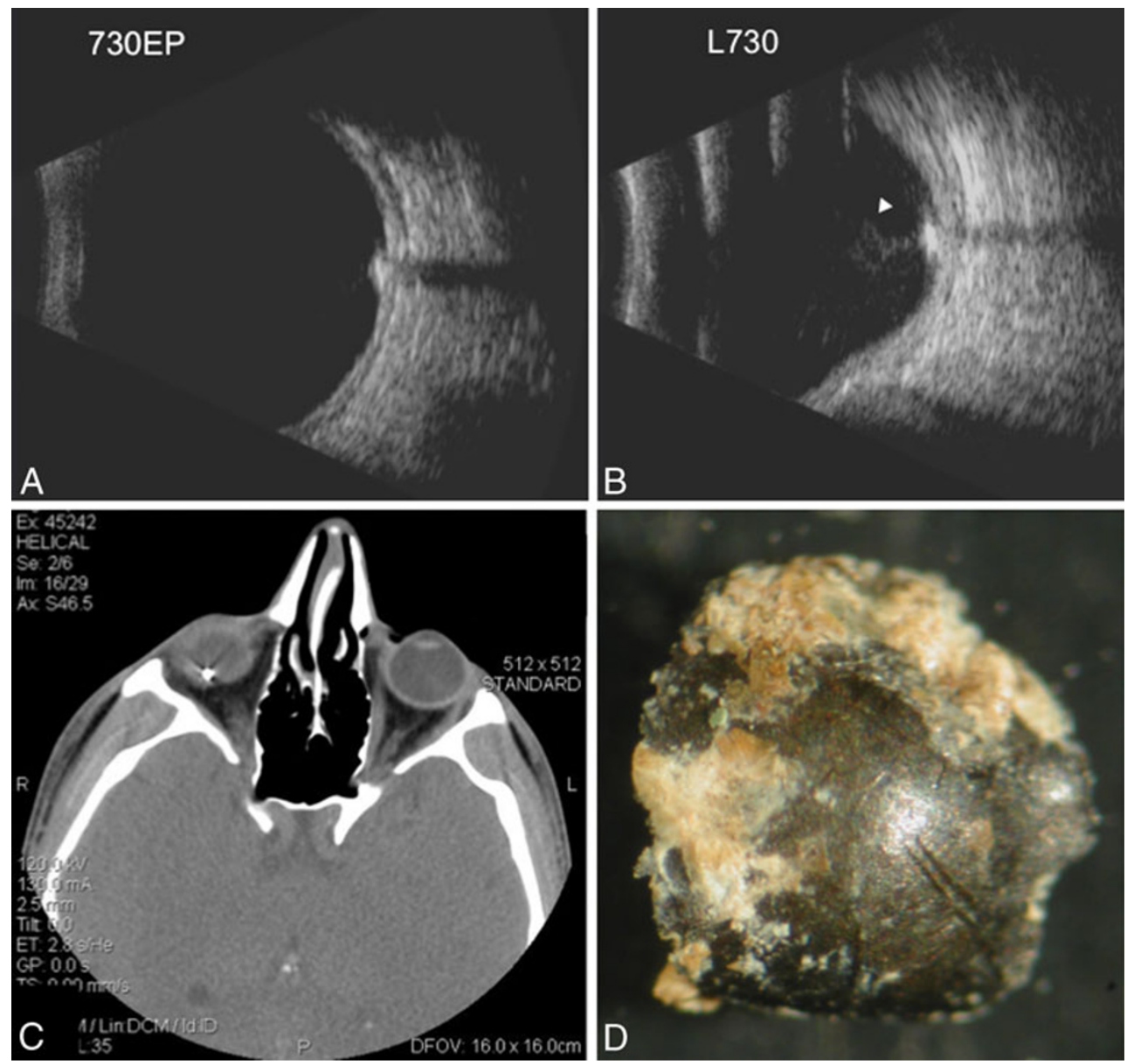

Fig. 2 B-scan ultrasound of the right eye shows an intraocular foreign body (IOFB) with shadowing behind the IOFB on the 7:30 equator posterior $(730 E P)$ and 7:30 longitudinal (L730) scans (a, b). There is moderate vitreous debris overlying the IOFB but no distinct track in

flow rate of $15 \mathrm{~L} / \mathrm{min}$, carrier gas flow rate of $1.04 \mathrm{~L} / \mathrm{min}$, and helium (He) gas flow rate of $4.3 \mathrm{ml} / \mathrm{min}$ (only in $\mathrm{He}$ mode). To evaluate which elements were present in the dissolved fragment, count rates for most common elements $(\sim 50)$ were monitored using a constant spray of the sample. During this qualitative portion of the analysis, only iron showed an extremely high count rate, while few elements gave slightly elevated count rates that were $\sim 10^{3} \times$ less than those for iron.

For the subsequent quantitative analysis, a method was set up for all elements that had shown elevated count rates. Data were quantified using a seven-point $(0,1,10,100$, $1,000,2,000$, and $5,000 \mathrm{ppb}(\mathrm{ng} / \mathrm{g}))$ calibration curve with external standards for $\mathrm{Mg}, \mathrm{Al}, \mathrm{Ca}, \mathrm{Fe}, \mathrm{Cu}, \mathrm{Zn}$, and $\mathrm{Pb}$. All data were acquired in He mode to remove interference from oxides, argides, and chlorides. For each sample, run data were acquired in triplicate and averaged. Internal standards introduced with the sample were used to correct for plasma instabilities. The National Institute of Standards and the vitreous (white arrow). c Axial CT scan confirms IOFB with metallic streaking artifact. d Gross photograph of metallic IOFB prior to inductively coupled mass spectrometry

Technology standard reference material was used to ensure elemental recovery of $>90 \%$.

\section{ICP-MS results}

The quantitative metallic composition derived from ICPMS analysis is summarized in Table 1. Approximately,

Table 1 Composition of metallic IOFB using inductively coupled mass spectrometry

\begin{tabular}{lclc}
\hline Element & Average (ppm) & Standard deviation & Total (\%) \\
\hline $\mathrm{Fe}$ & 389.32 & 12.29 & 97.33 \\
$\mathrm{Cu}$ & 0.11 & 0.04 & 0.03 \\
$\mathrm{Zn}$ & 0.77 & 0.80 & 0.19 \\
$\mathrm{Al}$ & 1.01 & NA & 0.25 \\
$\mathrm{Ca}$ & 3.37 & NA & 0.84 \\
$\mathrm{~Pb}$ & 0.01 & 0.01 & 0.00 \\
\hline
\end{tabular}


$97.3 \%$ of the metallic IOFB was identified as iron, measuring $389 \pm 12.3 \mathrm{ppm}$ ( \pm standard deviation). Other elements identified included copper $(0.11 \pm 0.04)$ and zinc $(0.77 \pm 0.80)$. Calcium and aluminum were also found in minimal quantities. Mercury, cadmium, and lead were not identified in any significant quantity.

\section{Electrophysiologic testing}

A full-field electroretinogram (ERG) was performed 2 months following surgery, as the patient's vision remained 20/50 despite successful anatomic reattachment and a normal foveal contour. Photoreceptor responses were severely abnormal for the right eye. Rods and darkadapted cone responses were severely subnormal. The scotopic bright flash a-wave responses were moderately subnormal, whereas the b-wave amplitudes were markedly subnormal, creating an electronegative configuration consistent with siderosis.

\section{Clinical course}

At the final 9-month follow-up, the patient's visual acuity improved to 20/30 OD and remained 20/20 OS. Slit lamp examination showed no evidence of anterior chamber or vitreous inflammation. The retina remained attached with good chorioretinal scarring at the IOFB impact site, which was supported by the scleral buckle. The retinal hemorrhage OS resolved, and no inflammation was observed. The ERG was repeated and the right eye again demonstrated decreased amplitudes and prolonged timing of both rodand cone-driven responses, though these were improved compared to the initial ERG.

\section{Discussion}

Occult penetrating globe injuries with retained IOFBs are a relatively infrequent occurrence [1-6] and may rarely present with chronic anterior or posterior segment inflammation $[2,5,6]$. Besides meticulous ophthalmic examination, B-scan ultrasound, ultrasound biomicroscopy, and CT scans are extremely useful in the diagnosis of occult IOFB injuries. ERG changes have been characterized in the context of siderosis associated with IOFB injuries $[1,3]$. In addition, retained copper IOFBs may result in acute [1] or chronic chalcosis [7-9]. In both siderosis and chalcosis, ERG changes may be partially reversible in some patients $[10,11]$.

Following PPV/IOFB extraction and retinal detachment repair, our patient's visual acuity was decreased despite a successful surgical outcome. Because of the mild anterior chamber inflammation and retinal hemorrhage observed OS, there was also suspicion for early sympathetic ophthalmia. For these reasons, electrophysiology was performed and revealed a reduction in rod and darkadapted cone responses in both eyes, albeit to a much greater degree OD.

The ERG findings were most consistent with siderosis OD and were partially reversible with repeat testing. Contralateral ERG abnormalities have not been reported in the context of siderosis, but fortunately, these changes completely reversed on repeat testing. For this reason, the patient was treated only with routine postoperative medications, and systemic immunosuppression was deferred. Prior reports analyzing the heavy metal content of IOFBs have used diagnostic X-ray spectrometry $[12,13]$ and the percentage copper content of an IOFB likely plays a role in the degree of copper dissemination and associated toxicity.

In one prior patient reported with a copper IOFB, an undiluted vitreous sample showed an inflammatory reaction consisting of $\mathrm{CD}^{+} \mathrm{T}$ lymphocytes and polymorphonuclear leukocytes [13]. Our patient demonstrated moderate inflammation prior to PPV/IOFB removal and the low-grade inflammation in the contralateral eye was suspicious for an autoimmune reaction. It is not clear whether this represented early sympathetic ophthalmia, but the inflammation OS completely resolved without medical therapy. We hypothesize that, following the initial IOFB injury, the intraocular inflammation was due to the toxic effects of the iron fragment. IOFB extraction led to a decrease in localized ocular inflammation and a resultant improvement in the ERG findings. The etiology of the contralateral eye inflammation is not clear; however, the mild anterior chamber inflammation in the contralateral eye had resolved at final follow-up.

ICP-MS is an extremely sensitive technique capable of detecting metal fragments approaching 1 part in $10^{12}$ (parts per trillion) and was extremely valuable in identifying iron as the predominant heavy metal in the IOFB; copper was found in minimal quantities. Reversal of ERG abnormalities has been described previously following IOFB extraction, and based on the patient's clinical course, the patient's visual prognosis remains favorable. He is under surveillance for the development of sympathetic ophthalmia given his occult penetrating globe injury.

\section{Conclusion}

In summary, ICP-MS and ERG accompanied PPV/IOFB retinal detachment repair in the successful management of this patient. The combination of these techniques was also instrumental in documenting the resolution of retinal 
changes, providing prognostic information, and may be valuable in the medical and surgical management of these complex cases.

Acknowledgements This research was supported by an unrestricted grant from the Research to Prevent Blindness (New York, NY) to the Casey Eye Institute, Oregon Health and Science University, and Emory Eye Center, Emory University School of Medicine.

Open Access This article is distributed under the terms of the Creative Commons Attribution Noncommercial License which permits any noncommercial use, distribution, and reproduction in any medium, provided the original author(s) and source are credited.

\section{References}

1. Welch RB (1975) Two remarkable events in the field of intraocular foreign body: (1) The reversal of siderosis bulbi. (2) The spontaneous extrusion of an intraocular copper foreign body. Trans Am Ophthalmol Soc 73:187-203

2. Duker JS, Fischer DH (1989) Occult plastic intraocular foreign body. Ophthalmic Surg 20:169-170

3. Weiss MJ, Hofeldt AJ, Behrens M, Fisher K (1997) Ocular siderosis. Diagnosis and management. Retina 17:105-108
4. Deramo VA, Shah GK, Baumal CR et al (1999) Ultrasound biomicroscopy as a tool for detecting and localizing occult foreign bodies after ocular trauma. Ophthalmology 106:301-305

5. Kaushik S, Ichhpujani P, Ramasubramanian A, Pandav SS (2008) Occult intraocular foreign body: ultrasound biomicroscopy holds the key. Int Ophthalmol 28:71-73

6. Stangos AN, Pournaras CJ, Petropoulos IK (2005) Occult anterior-chamber metallic fragment post-phacoemulsification masquerading as chronic recalcitrant postoperative inflammation. Am J Ophthalmol 139:541-542

7. Rosenthal AR, Marmor MF, Leuenberger P, Hopkins JL (1979) Chalcosis: a study of natural history. Ophthalmology 86:1956-1972

8. Rao NA, Tso MO, Rosenthal AR (1976) Chalcosis in the human eye. A clinicopathologic study. Arch Ophthalmol 94:1379-1384

9. Neubauer H (1979) The Montgomery Lecture 1979. Ocular metallosis. Trans Ophthalmol Soc U K 99:502-510

10. Kuhn F, Witherspoon CD, Skalka H, Morris R (1992) Improvement of siderotic ERG. Eur J Ophthalmol 2:44-45

11. Dayan MR, Cottrell DG, Mitchell KW (1999) Reversible retinal toxicity associated with retained intravitreal copper foreign body in the absence of intraocular inflammation. Acta Ophthalmol Scand 77:597-598

12. Neumann R, Belkin M, Loewenthal E, Gorodetsky R (1992) A longterm follow-up of metallic intraocular foreign bodies, employing diagnostic X-ray spectrometry. Arch Ophthalmol 110:1269-1272

13. Billi B, Lesnoni G, Scassa C, Giuliano MA, Coppe AM, Rossi T (1995) Copper intraocular foreign body: diagnosis and treatment. Eur J Ophthalmol 5:235-239 